\title{
Shut-Down of Type IX Protein Secretion Alters the Host Immune Response to Tannerella forsythia and Porphyromonas gingivalis
}

OPEN ACCESS

Edited by:

Ulvi Kahraman Gürsoy, University of Turku, Finland

Reviewed by:

Pinar Emecen-Huja, Medical University of South Carolina, United States

Keiji Nagano,

Health Sciences University of Hokkaido, Japan

*Correspondence: Christina Schäffer christina.schaeffer@boku.ac.at Oleh Andrukhov oleh.andrukhov@meduniwien.ac.at

${ }^{\dagger}$ These authors have contributed equally to this work

Specialty section:

This article was submitted to Microbiome in Health and Disease,

a section of the journal

Frontiers in Cellular and

Infection Microbiology

Received: 14 December 2021 Accepted: 24 January 2022

Published: 10 February 2022

Citation:

Braun ML, Tomek MB,

Grünwald-Gruber C, Nguyen $P Q$, Bloch S, Potempa JS, Andrukhov O and Schäffer C (2022) Shut-Down of

Type IX Protein Secretion Alters the Host Immune Response to Tannerella forsythia and

Porphyromonas gingivalis. Front. Cell. Infect. Microbiol. 12:835509.

doi: 10.3389/fcimb.2022.835509
Matthias L. Braun ${ }^{1 \dagger}$, Markus B. Tomek ${ }^{1 \dagger}$, Clemens Grünwald-Gruber ${ }^{2}$, Phuong Q. Nguyen ${ }^{3}$, Susanne Bloch ${ }^{1}$, Jan S. Potempa ${ }^{4}$, Oleh Andrukhov ${ }^{3 *}$ and Christina Schäffer ${ }^{\text {1* }}$

\begin{abstract}
Department of NanoBiotechnology, NanoGlycobiology Unit, Universität für Bodenkultur Wien, Vienna, Austria, ${ }^{2}$ Department of Chemistry, Institute of Biochemistry, Universität für Bodenkultur Wien, Vienna, Austria, ${ }^{3}$ Competence Center for Periodontal Research, University Clinic of Dentistry, Medical University of Vienna, Vienna, Austria, ${ }^{4}$ Oral Health and Systemic Disease Group, University of Louisville, Louisville, KY, United States
\end{abstract}

Tannerella forsythia and Porphyromonas gingivalis target distinct virulence factors bearing a structurally conserved C-terminal domain (CTD) to the type IX protein secretion system (T9SS). The T9SS comprises an outer membrane translocation complex which works in concert with a signal peptidase for CTD cleavage. Among prominent T9SS cargo linked to periodontal diseases are the TfsA and TfsB components of T. forsythia's cell surface (S-) layer, the bacterium's BspA surface antigen and a set of cysteine proteinases (gingipains) from $P$. gingivalis. To assess the overall role of the bacterial T9SS in the host response, human macrophages and human gingival fibroblasts were stimulated with T. forsythia and $P$. gingivalis wild-type bacteria and T9SS signal peptidase-deficient mutants defective in protein secretion, respectively. The immunostimulatory potential of these bacteria was compared by analyzing the mRNA expression levels of the pro-inflammatory mediators IL-6, IL-8, MCP-1 and TNF- $\alpha$ by qPCR and by measuring the production of the corresponding proteins by ELISA. Shot-gun proteomics analysis of $T$. forsythia and $P$. gingivalis outer membrane preparations confirmed that several CTD-bearing virulence factors which interact with the human immune system were depleted from the signal peptidase mutants, supportive of effective T9SS shut-down. Three and, more profoundly, 16 hours post stimulation, the T. forsythia T9SS mutant induced significantly less production of cytokines and the chemokine in human cells compared to the corresponding parent strain, while the opposite was observed for the $P$. gingivalis T9SS mutant. Our data indicate that T9SS shut-down translates into an altered inflammatory response in periodontal pathogens. Thus, the T9SS as a potential novel target for periodontal therapy needs further evaluation. 


\section{INTRODUCTION}

Oral health is characterized by the symbiotic interaction between the oral microbiota and the human host. Its disturbance by environmental or genetic factors leads to microbial dysbiosis and increases the risk of oral diseases, particularly periodontitis (Marsh and Zaura, 2017). Periodontitis is an inflammatory biofilm disease of the tooth-supporting tissues characterized by a dysbiotic state and the prevalence of the "red complex" of Gram-negative, anaerobic pathogens-Porphyromonas gingivalis, Tannerella forsythia and Treponema denticola (Holt and Ebersole, 2005; Socransky and Haffajee, 2005; Griffen et al., 2012). One of these bacteria, $P$. gingivalis, is considered as a keystone pathogen and can subvert the host immune response, disrupting the host-microbe homeostasis in the oral cavity and promoting a dysbiotic state, even when present at low quantities (Hajishengallis and Lamont, 2012).

The "red complex" bacteria interfere with metabolic and physiological functions of the host through virulence factors (Lasica et al., 2017; Gorasia et al., 2020). P. gingivalis and T. forsythia secrete distinct virulence factors across the outer membrane (OM) using the type IX secretion system (T9SS), which is regarded as an essential determinant of pathogenicity in periodontal diseases (Tomek et al., 2014). The T9SS seems to be characteristic of the Fibrobacteres-Chlorobi-Bacteroidetes superphylum to which $T$. forsythia and $P$. gingivalis are affiliated (Lasica et al., 2017). The T9SS machinery is composed of at least 18 essential protein components, of which orthologs exist in P. gingivalis and T. forsythia (Lasica et al., 2017; Lauber et al., 2018). These components build up a complex that translocates proteins possessing a structurally conserved carboxy-terminal domain (CTD) via the OM. The "classical" CTD is composed of 40-70 variable amino acid residues that possess an Ig-like fold (de Diego et al., 2016). The 3D structure of the CTD serves as a recognition element of proteins for the T9SS. After protein translocation to the surface, CTD-cleavage is catalyzed by a C-terminal signal peptidase named PG0026 (PorU) and Tanf_02580 in P. gingivalis W83 and T. forsythia ATCC 43037, respectively (Tomek et al., 2014; Lasica et al., 2016), components of the attachment complex built of PorQ, PorU, PorV and PorZ (Nguyen et al., 2007; Veith et al., 2009; Sato et al., 2013; Lauber et al., 2018). T9SS cargo proteins are either released to the environment (Lasica et al., 2017) or stay associated with the bacterial surface, predictably anchored into the $\mathrm{OM}$ by a glycoconjugate of so far unknown structure that is attached to the C-terminal residue (Veith et al., 2020).

In $P$. gingivalis, the gingipains-RgpA, RgpB, and Kgp-are intensely investigated cysteine proteases carrying a "classical" CTD for secretion via the T9SS; they have a myriad of roles in periodontitis. Gingipains cause hypo-responsiveness of several components of innate immunity like epithelial cells, macrophages, and neutrophils (Stathopoulou et al., 2009; Wilensky et al., 2015; Sochalska and Potempa, 2017), resulting in impaired bacterial clearance and a dysbiotic state. Furthermore, they degrade host cytokines and chemokines resulting in downregulation of the host response in the form of reduced inflammation (Stathopoulou et al., 2009; Stathopoulou et al., 2010). While gingipains are mainly attached to the surface of the OM, they may also be partially released in a soluble form into the extracellular milieu (Rangarajan et al., 1997). It was shown that a C-terminally truncated form of RgpB is no longer attached to the OM demonstrating the importance of the CTD signal for export and cell attachment (Seers et al., 2006).

Among prominent virulence proteins of $T$. forsythia which are equipped with a "classical" CTD for targeting to the T9SS are, for instance, the two heavily glycosylated surface (S-) layer proteins TfsA and TfsB (Sabet et al., 2003; Lee et al., 2006; Sakakibara et al., 2007; Sekot et al., 2011; Posch et al., 2012), BspA surface antigen (Sharma et al., 1998), and hemagglutinin (Murakami et al., 2002). The TfsA and TfsB proteins selfassemble into a $2 \mathrm{D}$ crystalline layer around T. forsythia cells; this S-layer mediates the adherence of the bacterium to the human gingival epithelium (Mishima and Sharma, 2011) and, at the early stage of infection, delays the immune response of human gingival fibroblasts (hGFBs) and macrophages (Sekot et al., 2011). BspA, on the other hand, is known to activate the host response in monocytes and epithelial cells through a TLR-2 dependent mechanism (Hajishengallis et al., 2002; Onishi et al., 2008). Furthermore, a set of six secretory proteases of T. forsythia cleave host proteins such as collagen (Ksiazek et al., 2015) and degrade complement proteins and the antimicrobial peptide LL37, which may contribute to virulence through evading innate immunity (Koneru et al., 2017). Of note, these proteases bear a nearly identical CTD that ends with a Lys-Leu-Ile-Lys-Lys motif (KLIKK), but share very limited sequence similarity with the "classical" CTD (Ksiazek et al., 2015).

Considering the link between CTD-proteins and virulence, it is likely that blocking of their OM-export in T. forsythia and $P$. gingivalis alters the pathogens' ability to induce a host response. Thus, the purpose of this study was to assess, if and in which aspects shut-down of the T9SS in T. forsythia and P. gingivalis influences the elicitation of a cellular response of human macrophages and hGFBs-both known for their roles in the pathogenesis of periodontitis. Specifically, along with the parent strains we used mutants of $T$. forsythia and $P$. gingivalis with a deletion in the T9SS signal peptidase gene resulting in a secretion defective phenotype. Using shot-gun proteomics, we first investigated the bacterias' OM proteome for the presence of key CTD-proteins of known virulence potential to assess the efficiency of the T9SS shut-down and then, challenged the two human cell types with the different bacterial species for up to 16 hours and determined the production of different inflammatory mediators using qPCR and ELISA. Specifically, we investigated the production of tumor necrosis factor (TNF)- $\alpha$, interleukin (IL)- 8 , and IL-6 in U937 macrophages and of IL-6, IL-8, and monocyte chemoattractant protein (MCP)- 1 in hGFBs. IL- 6 and TNF- $\alpha$ are involved in regulating the immune reactivity of acute-phase proteins and in recruiting of lymphocytes to inflamed tissues (Charlie-Silva et al., 2019). IL-8 and MCP-1 are strong chemoattractants and stimulate the migration of leukocytes to sites of infection (Baggiolini and Clark-Lewis, 1992; Deshmane et al., 2009). These inflammatory mediators are a substantial part 
of the host defense; insufficient cytokine production leads to an impaired bacterial elimination and development of a dysbiotic state (Olsen and Hajishengallis, 2016), whereas an excessive response might cause collateral tissue damages (Cekici et al., 2014).

\section{MATERIALS AND METHODS}

\section{Bacterial Strains and Growth Conditions}

The T. forsythia ATCC 43037 type strain (wild-type) and $P$. gingivalis BAA-308/W83 (wild-type) were obtained from the American Type Culture Collection (ATCC, Manassas, VA, USA). Deletion mutants of the signal peptidase gene encoding PorU (PG0026) of P. gingivalis (Lasica et al., 2016) (henceforth abbreviated $\triangle P G 0026$ ) and the T. forsythia ortholog Tanf_02580 (henceforth abbreviated $\Delta$ Tanf_02580) (Tomek et al., 2014) were available in our laboratory. T. forsythia wild-type and $\Delta$ Tanf_02580 were grown anaerobically in brain-heart-infusion (BHI) broth with supplements, at $37^{\circ} \mathrm{C}$ for 7 days as published previously (Tomek et al., 2014). P. gingivalis wild-type and $\triangle P G 0026$ were grown anaerobically in enriched tryptic soy broth (ETSB) at $37^{\circ} \mathrm{C}$ for 3 days (Tada et al., 2017). Bacteria were harvested by centrifugation at $5000 \mathrm{~g}$ for $20 \mathrm{~min}$ at $4^{\circ} \mathrm{C}$ and washed once with the respective growth medium.

For stimulation of human cells, bacterial pellets were resuspended in RPMI 1640 medium (Invitrogen, Waltham, MA, USA) and the optical density at $600 \mathrm{~nm}\left(\mathrm{OD}_{600}\right)$ was set to 1 with medium. A correlation between $\mathrm{OD}_{600}$ values of 1.0 and colony forming units (CFU) per milliliter of culture of the different bacteria and mutants included in this study was determined by dilution plating and colony counting (three biological replicates with three technical replicates, each), with $\mathrm{OD}_{600}=1.0$ corresponding to $3 \times 10^{8} \mathrm{CFU}$ of $T$. forsythia wildtype, $5 \times 10^{8} \mathrm{CFU}$ of $T$. forsythia $\triangle$ Tanf_02580, $1 \times 10^{9} \mathrm{CFU}$ of $P$. gingivalis wild-type, and $1 \times 10^{9} \mathrm{CFU}$ of $P$. gingivalis $\triangle P G 0026$, respectively. Bacterial suspensions were immediately used for further processing.

\section{Outer Membrane Isolation}

For the isolation of outer membranes, $1 \mathrm{~g}$ of wet pellet of $T$. forsythia wild-type, $\Delta$ Tanf_02580, $P$. gingivalis wild-type, and $\triangle P G 0026$, respectively, was resuspended in $25 \mathrm{~mL}$ of phosphatebuffered saline (PBS) and disrupted by sonication on ice for 15 min $/ 50 \%$ power $/ 30$ s duty cycle, using a Branson Ultrasonics Sonifier $^{\mathrm{TM}}$ (Branson, Brookfield, CT, USA). The OM isolation essentially followed a protocol published for $T$. forsythia (Komatsuzawa et al., 2002; Shimotahira et al., 2013). Briefly, undisrupted bacteria were removed and membrane fractions collected by ultracentrifugation at $100,000 \mathrm{~g}$ for $1 \mathrm{~h} / 4^{\circ} \mathrm{C}$ (Ti70.1 rotor; Beckman, Brea, CA, USA) and washed with PBS. The pellet was resuspended in $5 \mathrm{~mL}$ of $1 \%(w / v)$ sodium lauryl sarcosine (Sigma-Aldrich, St. Louis, MO, USA) and the insoluble $\mathrm{OM}$ fraction was re-dissolved in $500 \mu \mathrm{L}$ of $1 \%(w / v)$ sodium dodecyl sulphate (SDS) $(w / v)$. The OM isolation was performed in triplicate, each, and the preparations were analyzed by SDSPAGE using 10\% gels according to Laemmli (Laemmli, 1970) and separated protein bands were stained with Coomassie Brilliant Blue G250 (CBB). Protein concentrations of the OM preparations were determined by the Bradford assay (Bradford, 1976).

\section{Shot-Gun Proteomics}

For shot-gun proteomics, the SDS-dissolved OM preparations were digested in-solution. The proteins were S-alkylated with iodoacetamide and digested with trypsin (Promega, Madison, WI, USA) as described elsewhere (Gundry et al., 2009). The digested samples were loaded on a BioBasic C18 column (BioBasic-18, 150 × $0.32 \mathrm{~mm}, 5 \mu \mathrm{m}$; Thermo Fisher Scientific, Waltham, MA, USA) using $80 \mathrm{mM}$ ammonium formate buffer at a flow rate of $6 \mu \mathrm{L} \mathrm{min}{ }^{-1}$ as solvent $A$. A gradient from $5 \%$ solvent B ( $80 \%$ acetonitrile) in solvent A to $40 \%$ solvent B over 45 min was applied, followed by a 15 -min gradient from $40 \%$ solvent B to $95 \%$ solvent B to facilitate the elution of large peptides. Detection was performed with QTOF MS (Bruker maXis 4G; Bruker, Billerica, MA, USA) equipped with the standard ESI source in positive ion, DDA mode (switching to MSMS mode for eluting peaks). MS-scans were recorded (range: 150-2200 Da) and the six highest peaks were selected for fragmentation. For instrument calibration, ESI calibration mixture (Agilent, Santa Clara, CA, USA) was used. The analysis files were converted to mgf files (using Data Analysis, Bruker), which are suitable for performing an MS/MS ion search with ProteinScape (Bruker, MASCOT embedded). The files were searched against the UniProt database.

\section{Isolation and Growth Conditions of Human Monocytes and Human Gingival Fibroblasts}

The U937 monocytic cell line was purchased from the ATCC and cultured in RPMI 1640 medium, supplemented with 10\% ( $v / v)$ fetal bovine serum (FBS) and penicillin (100 $\left.\mathrm{U} \mathrm{mL}^{-1}\right)$ streptomycin $\left(100 \mu \mathrm{g} \mathrm{mL}^{-1}\right)$ (Pen-Strep) at $37^{\circ} \mathrm{C}$ in a humidified atmosphere containing $5 \% \mathrm{CO}_{2}$ (Friedrich et al., 2015).

hGFBs were isolated from the gingival tissue of periodontally and systemically healthy individuals (Sekot et al., 2011). Gingival tissue was cut off with a scalpel, placed into Dulbecco's Modified Eagle's Medium (DMEM; Invitrogen) supplemented with $10 \%$ FBS, Pen-Strep, shredded into small pieces, and incubated at $37^{\circ} \mathrm{C}$ and $5 \% \mathrm{CO}_{2}$ for cell outgrowth.

\section{Stimulation of Human Macrophages and Human Gingival Fibroblasts With Bacteria}

Prior to stimulation with bacteria, U937 monocytes were differentiated into macrophages as described previously (Sekot et al., 2011; Friedrich et al., 2015). Briefly, three milliliters of cell suspension at a concentration of $10^{6}$ cells $\mathrm{mL}^{-1}$ were added per well of a 6-well plate and cells were stimulated with phorbol 12myristate 13-acetate (Sigma-Aldrich) at a concentration of $0.2 \mu \mathrm{g}$ $\mathrm{mL}^{-1}$ for 72 hours.

Adherent macrophages were gently scraped, counted and seeded in a 24 -well plate at a density of $2 \times 10^{5}$ cells/well in 0.5 $\mathrm{mL}$ of RPMI 1640 medium supplemented with $10 \%$ FBS and $1 \%$ 
Pen-Strep. hGFBs were seeded at a density of $5 \times 10^{4}$ cells/well in $0.5 \mathrm{~mL}$ of DMEM containing the same supplements. After 24 hours, the media were discarded, cells were rinsed once with PBS, subsequently, $0.5 \mathrm{~mL}$ of the respective medium without FBS, but containing Pen-Strep was added, and cells were exposed to the different bacterial stimuli at a multiplicity of infection (MOI) of 50 (Sekot et al., 2011). Six and five independent experiments for U937 and hGFB, respectively, with four technical replicates, each, were performed and FBS-free medium containing PenStrep without bacteria served as a negative control. Stimulation was done for 3 hours and 16 hours, at $37^{\circ} \mathrm{C}$ and $5 \% \mathrm{CO}_{2}$.

\section{MTT Cell Viability Assay}

After cell stimulation, $100 \mu \mathrm{L}$ of 3-(4,5-dimethylthiazol-2-yl)-2,5diphenyltetrazolium bromide (MTT) dye $\left(5 \mathrm{mg} \mathrm{mL}^{-1}\right.$ in PBS) was added to the cells and the plates were incubated at $37^{\circ} \mathrm{C}$ for 2 hours (Vistica et al., 1991). Subsequently, the medium was discarded and $500 \mu \mathrm{L}$ of dimethylsulfoxide were added to each well and the plates were shaken to facilitate dissolving of formazan crystals. Controls were performed in which each bacterium was solely added. $\mathrm{OD}_{570}$ values were measured on a Spectramax Plus micro-plate reader (Molecular Devices, Sunnyvale, CA, USA). The MTT assay was performed in five replicates.

\section{Gene Expression Analysis of Inflammatory Mediators}

At the end of the stimulation, the cell supernatant was collected and aspirated for ELISA quantification of secreted inflammatory mediators. Adherent cells were washed with PBS followed by detachment from the wells with a cell scraper (macrophages) or accutase (hGFBs; Thermofisher Scientific) and used for geneexpression analysis.

Isolation of mRNA, transcription into cDNA, and qPCR was performed using the TaqMan ${ }^{\circledR}$ Gene Expression Cells-to-CT ${ }^{\mathrm{TM}}$ kit (Ambion/Applied Biosystems, Foster City, CA, USA) (Behm et al., 2019; Blufstein et al., 2019). The target genes were amplified using the following primers (all Applied Biosystems): TNF- $\alpha$, Hs99999043_m1; IL-6, Hs00985639_m1; IL-8, Hs00174103_m1; MCP-1, Hs00234140; GAPDH, Hs99999905_m1. qPCR was performed in paired reactions using the ABI StepOnePlus device with the following setting: $10 \mathrm{~min}$ at $95^{\circ} \mathrm{C}, 50$ cycles at $95^{\circ} \mathrm{C}$ for 15 seconds and $60^{\circ} \mathrm{C}$ for 60 seconds. $\mathrm{C}_{\mathrm{t}}$ values were determined for each gene and the expression of the target gene was determined by the $2^{-\Delta \Delta C t}$ method, where $\Delta \Delta \mathrm{C}_{t}=\left(\mathrm{C}_{\mathrm{t}}^{\text {target }}-\mathrm{C}_{\mathrm{t}}^{\mathrm{GAPDH}}\right)$ sample $\left(\mathrm{C}_{t}^{\text {target }}-\mathrm{C}_{t}^{\mathrm{GAPDH}}\right)$ control. Cells, which were not treated with bacteria, served as control. For U937 macrophages, expression of IL-6, IL-8 and TNF- $\alpha$ was analyzed, for hGFBs, IL-6, IL-8 and MCP-1.

\section{Determination of Secreted Cytokines and Chemokines by ELISA}

The concentration of the inflammatory mediators IL-6, IL-8, TNF- $\alpha$, and MCP-1 in conditioned media, i.e., cell culture supernatant after stimulation, was determined using ELISA Ready-SET-Go kits (eBioscience, Santa Clara, CA, USA) according to the manufacturer's protocol.

\section{Statistical Analysis}

ANOVA's statistic for the repeated measure followed by the post-hoc LSD test for pairwise comparisons was used to analyze statistical differences. Statistical analysis was performed using SPSS 24.0 software (IBM, Armonk, NY, USA). All data are expressed as mean \pm standard error of the mean (s.e.m.). Significant statistical differences were considered at $P<0.05$.

\section{RESULTS}

\section{CTD-Proteins Are Depleted From the Outer Membrane of a $T$. forsythia and $P$. gingivalis T9SS Signal Peptidase Mutant}

Prior to analyzing the influence of the T9SS shut-down on the immune response of U937 macrophages and hGFBs to T. forsythia and $P$. gingivalis challenges, the efficiency of CTD-protein depletion from the OM in the signal peptidase mutants $\Delta T a n f \_02580$ (Tomek et al., 2014) and $\triangle P G 0026$ was investigated.

First, SDS-PAGE analysis of the OM preparations from $T$. forsythia wild-type, $\Delta$ Tanf_02580, $P$. gingivalis wild-type, and $\triangle P G 0026$ revealed different $\mathrm{CBB}$-stained banding patterns of the respective parent and mutant strains, indicative of the absence of proteins in the molecular-mass regions of known CTD-proteins (compare with Tables 1, 2) in the T9SS signal peptidase mutants (Supplementary Figure 1).

Next, the OM preparations from $T$. forsythia wild-type, $\triangle$ Tanf_02580, P. gingivalis wild-type, and $\triangle P G 0026$ were subjected to shot-gun proteomics. Specifically, we performed a closer inspection for the presence of known CTD-proteins in order to assess the efficiency of their translocation blockage via deletion of the T9SS signal peptidase genes PG0026 and Tanf_02580, respectively.

According to MS analysis of peptide fingerprints, in the OM preparation of the $\Delta$ Tanf_02580 mutant (Table 1), several known CTD-bearing virulence factors were no longer detectable. Among these were the surface antigen BspA (Tanf_04820), a possible hemagglutinin/hemolysin (Tanf_06020) and the S-layer protein TfsB (Tanf_03375), while the second S-layer protein, TfsA (Tanf_03370) was massively reduced. It is likely that due to the high cellular abundance of the S-layer proteins residual amounts of TfsA might originate from cross-contamination with periplasmic content during cell fractionation. These results not only confirm but also expand our previous analysis of the $\Delta$ Tanf_02580 mutant (Tomek et al., 2014) and corroborate data showing that other T. forsythia CTD-proteins (Veith et al., 2009) are missing in the OM fraction of the signal peptidase mutant but are present in that of the T. forsythia wild-type. These included IgG Fc binding domain-containing proteins (Tanf_00065, Tanf_11855), a bacterial group 2 Ig-like protein (Tanf_03310), a conserved repeat protein (Tanf_08920), as well as hypothetical proteins (Tanf_08965, Tanf_02330, Tanf_02425). Notably, in contrast to these proteins bearing a "classical" CTD, the KLIKK proteases of $T$. forsythia are secreted directly into the extracellular medium, and, thus, cannot be detected in either the wild-type bacterium or the T9SS signal peptidase mutant (Veith et al., 2009). 
TABLE 1 | CTD proteins detected by MS in the OM from T. forsythia ATCC 43037 wild-type (wt Tf) and T9SS signal peptidase-deficient mutant ( $\Delta$ Tanf_02580).

\begin{tabular}{|c|c|c|c|c|c|c|}
\hline \multirow[t]{2}{*}{ Protein name } & \multicolumn{2}{|c|}{ Protein } & \multicolumn{2}{|c|}{ MASCOT score* } & \multicolumn{2}{|c|}{ Sequence coverage $(\%)^{\star \star}$} \\
\hline & Locus tag & Amino acids & wt Tf & $\Delta T a n f \_02580$ & wt $T f$ & $\Delta T a n f \_02580$ \\
\hline Surface antigen BspA & Tanf_04820 & 1156 & 784.7 & 0 & 19.1 & 0 \\
\hline Possible hemagglutinin & Tanf_06020 & 1252 & 134.3 & 0 & 5.2 & 0 \\
\hline S-layer protein TfsA & Tanf_03370 & 1166 & 4002 & 350 & 49.9 & 7.8 \\
\hline S-layer protein TfsB & Tanf_03375 & 1347 & 3415 & 0 & 46,7 & 0 \\
\hline IgG Fc binding domain-containing protein & Tanf_00065 & 598 & 1228 & 0 & 29.1 & 0 \\
\hline IgG Fc binding domain-containing protein & Tanf_11855 & 613 & 1235 & 0 & 24.3 & 0 \\
\hline Bacterial group 2 lg-like protein & Tanf_03310 & 376 & 241.2 & 0 & 12 & 0 \\
\hline Conserved repeat protein & Tanf_08920 & 764 & 212.3 & 0 & 5.8 & 0 \\
\hline Hypothetical protein, uncharacterized & Tanf_08965 & 1562 & 670 & 0 & 9.6 & 0 \\
\hline Hypothetical protein, uncharacterized & Tanf_02330 & 1830 & 477.5 & 0 & 8.1 & 0 \\
\hline Hypothetical protein, uncharacterized & Tanf_02425 & 1457 & 976.4 & 0 & 19.4 & 0 \\
\hline
\end{tabular}

${ }^{*}$ Results from one representative preparation from three biological replicates in terms of MASCOT scores are shown. ** Only peptides covering more than $5 \%$ of the sequence were considered.

In the case of $P$. gingivalis, peptide fingerprints of the dominant $P$. gingivalis Cys-proteases RgpA (PG2024), RgpB (Arg-specific; PG0506) and Kgp (Lys-specific; PG1844) as well as of hemagglutinin HagA (PG1837), immunoreactive antigen PG91 (PG2102) and peptidylarginine deiminase (PG1424) were found exclusively in the OM preparation form the $P$. gingivalis wild-type (Table 2).

\section{Effect of $T$. forsythia and $P$. gingivalis Wild- Type and the $\triangle T a n f 02580$ and $\triangle P G 0026$ Mutant on the Viability of U937 Macrophages and Gingival Fibroblasts}

The effect of the different wild-type bacteria and mutants on the viability of U937 macrophages and hGFBs was investigated prior to determining the immunostimulatory potential of $T$. forsythia and $P$. gingivalis wild-type versus the respective T9SS signal peptidase deficient mutant.

All tested T. forsythia and P. gingivalis species promoted the viability of U937 macrophages, three hours and 16 hours post stimulation (Figures 1A, B). No differences in the viability of the macrophages were observed between challenges with wild-type species versus the corresponding T9SS-deficient mutant. In contrast, none of the tested bacteria had a significant influence on the viability of hGFBs after both three hours and 16 hours post stimulation (Figures 1C, D). Bacteria alone did not show a measurable reactivity with the MTT reagent under the chosen experimental conditions.

\section{Effect of $T$. forsythia Wild-Type and the $\Delta$ Tanf_02580 Mutant on the Host Response of U937 Macrophages}

The effect of T. forsythia wild-type and $\triangle$ Tanf_02580 on the gene expression levels of TNF- $\alpha$, IL-6, and IL-8, and on the production of the corresponding proteins in U937 macrophages is shown in Figure 2. Both T. forsythia species increased the gene expression of all investigated inflammatory mediators (Figure 2A). Three hours post stimulation, T. forsythia wild-type induced significantly higher expression levels of all genes compared to the $\Delta$ Tanf_02580 mutant. After 16 hours, neither T. forsythia strain had an effect on TNF- $\alpha$ gene expression, while both, wild-type and mutant, stimulated IL-8 gene expression by a similar degree. Gene expression of IL-6 after 16 hours was below the detection limit in the bacteria-treated groups. Sixteen hours post stimulation, both $T$. forsythia species increased the production of all investigated inflammatory mediators (Figure 2B), but no significant differences between $T$. forsythia wild-type and the T9SS signal peptidase mutant were observed.

\section{Effect of T. forsythia Wild-Type and the $\Delta$ Tanf_02580 Mutant on the Host Response of Human Gingival Fibroblasts}

Figure 3 shows the effect of $T$. forsythia wild-type and $\Delta$ Tanf_02580 on the gene expression levels of IL-6, IL-8 and $\mathrm{MCP}-1$, and the production of the corresponding proteins in hGFBs. Both T. forsythia species induced a significant increase in

TABLE 2 | CTD proteins detected by MS in the OM from P. gingivalis W83 wild-type (wt Pg) and T9SS signal peptidase-deficient mutant ( $\triangle P G 0026)$.

\begin{tabular}{|c|c|c|c|c|c|c|}
\hline Protein name & \multicolumn{2}{|c|}{ Protein } & \multicolumn{2}{|c|}{ MASCOT score* } & \multicolumn{2}{|c|}{ Sequence coverage $(\%)^{\star \star}$} \\
\hline Arginine-specific protease RgpA & PG2024 & 1706 & 2140 & 0 & 23 & 0 \\
\hline Lysine-specific cysteine proteinase Kgp & PG1844 & 1727 & 2145 & 0 & 22 & 0 \\
\hline Hemagglutinin protein HagA & PG1837 & 2105 & 908 & 0 & 13 & 0 \\
\hline Immunoreactive 61-kDa antigen PG91 & PG2102 & 540 & 332 & 0 & 13 & 0 \\
\hline
\end{tabular}

${ }^{*}$ Results from one representative preparation from three biological replicates in terms of MASCOT scores are shown. ${ }^{*}$ Only peptides covering more than $5 \%$ of the sequence were considered. 
A

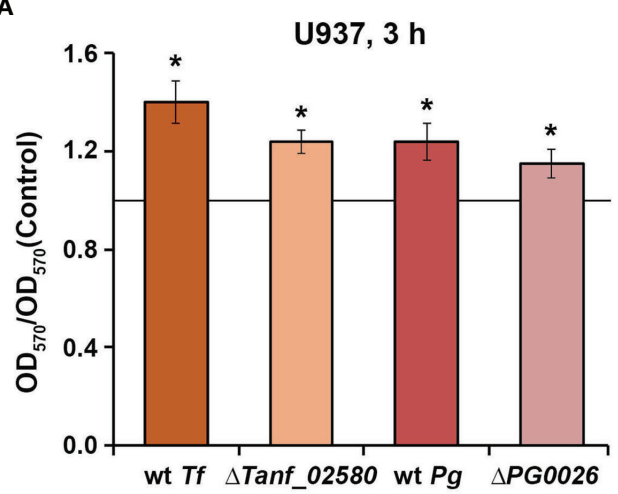

C

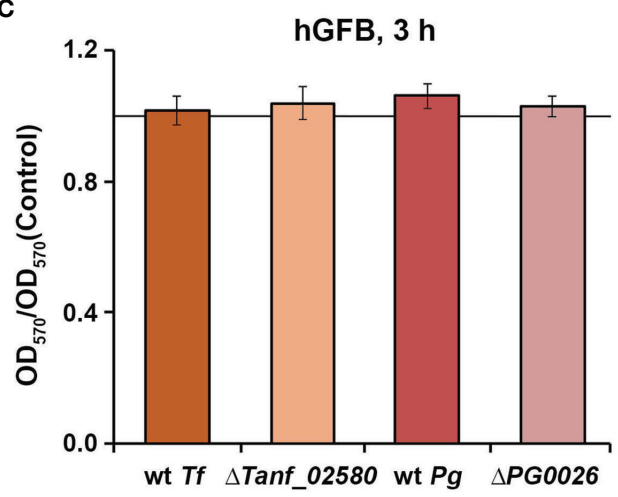

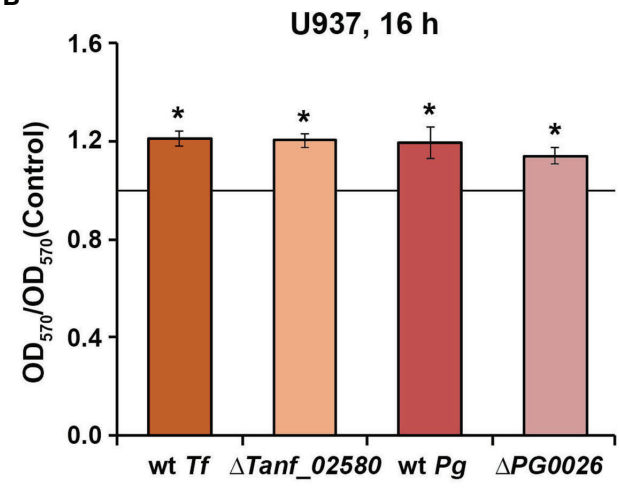

D

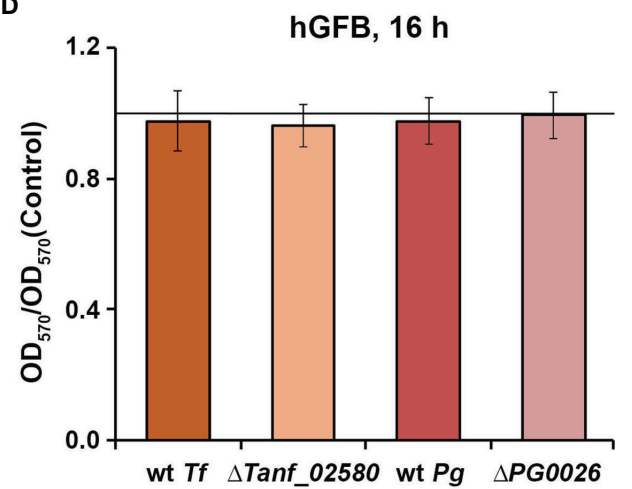

FIGURE 1 | Viability of U937 macrophages (A, B) and hGFBs (C, D) upon stimulation with T. forsythia wild-type (wt Tf), $\Delta$ Tanf_02580, P. gingivalis wild-type (wt $P g$ ), and $\triangle P G 0026$ at $\mathrm{MOI} 50$ for $3 \mathrm{~h}$ or $16 \mathrm{~h}$. Cell viability was measured with the MTT assay. $\mathrm{OD}_{570}$ values were normalized to those measured for non-stimulated cells (control). Data are presented as mean \pm s.e.m. of five independent experiments. ${ }^{*}-$ Significantly different from control (a continues horizontal line), with $P<0.05$.

the gene expression of IL-6, IL-8, and MCP-1, three hours and 16 hours post stimulation. T. forsythia wild-type induced significantly higher gene expression levels than the $\Delta$ Tanf_02580 mutant. Also, both T. forsythia species increased the production of all investigated cytokines compared to the control (Figure 3B), but, again, T. forsythia wild-type induced a significantly higher protein production than the T9SS signal peptidase mutant.

\section{Effect of $P$. gingivalis Wild-Type and the $\triangle P G 0026$ Mutant on the Host Response of U937 Macrophages}

The gene expression levels of TNF- $\alpha$, IL-6, and IL-8 in U937 macrophages upon stimulation with $P$. gingivalis wild-type and the $\triangle P G 0026$ mutant, and the levels of the corresponding proteins are shown in Figure 4. Three hours post stimulation, the expression of TNF- $\alpha$, IL- 6 , and IL- 8 was significantly increased in the $P$. gingivalis wild-type, whereas the $\triangle P G 0026$ mutant significantly enhanced the expression of TNF- $\alpha$ and IL8, but not of IL-6 (Figure 4A). No significant difference between the response of U937 macrophages to the two different $P$. gingivalis species was observed. Sixteen hours post stimulation, with both $P$. gingivalis species, the gene expression levels of TNF$\alpha$ and IL- 8 were similar to those in the unstimulated control. IL- 6 expression after stimulation was below the detection limit
(Figure 4A). After 16 hours, the levels of TNF- $\alpha$ and IL-8 in the conditioned media of $P$. gingivalis wild-type-treated macrophages were significantly lower than those in macrophages treated with the $\triangle P G 0026$ mutant and the control group (Figure 4B). IL-6 protein in the conditioned media of $P$. gingivalis wild-type-treated macrophages was below the detection limit of the ELISA kit. Stimulation with the $\triangle P G 0026$ mutant resulted in a significantly higher amount of IL- 8 and IL- 6 in the conditioned media compared to the control group, whereas TNF- $\alpha$ production was not affected.

\section{Effect of $\boldsymbol{P}$. gingivalis Wild-Type and the $\triangle P G 0026$ Mutant on the Host Response of Human Gingival Fibroblasts}

The effect of $P$. gingivalis wild-type and $\triangle P G 0026$ on the gene expression levels of IL-6, IL-8, and MCP-1 in hGFBs, and the levels of corresponding proteins in conditioned media are presented in Figure 5. Both $P$. gingivalis species induced a significant increase in the gene expression of IL-6, IL-8, and MCP-1 three hours post stimulation, but no difference between the two $P$. gingivalis species was observed. Sxiteen hours post stimulation, only the $\triangle P G 0026$ mutant induced significantly higher gene expression levels of all investigated inflammatory mediators, which were also significantly higher than those in $P$. gingivalis wild-type-treated cells 
A
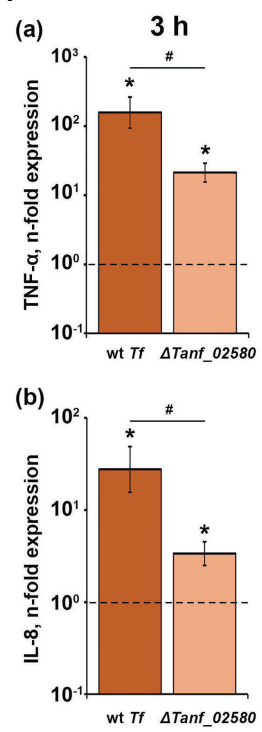

(c)

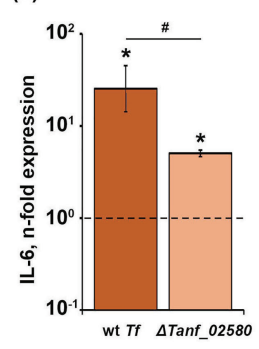

B

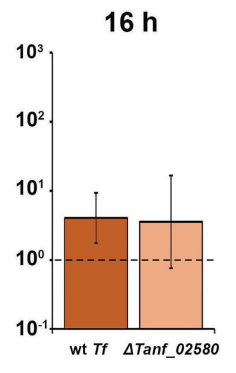

(a)

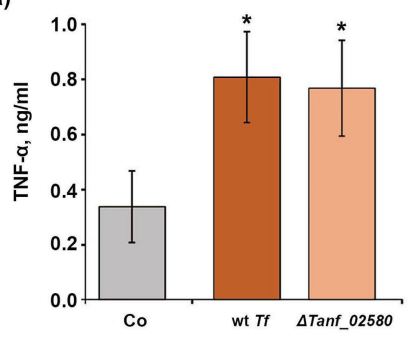

(b)
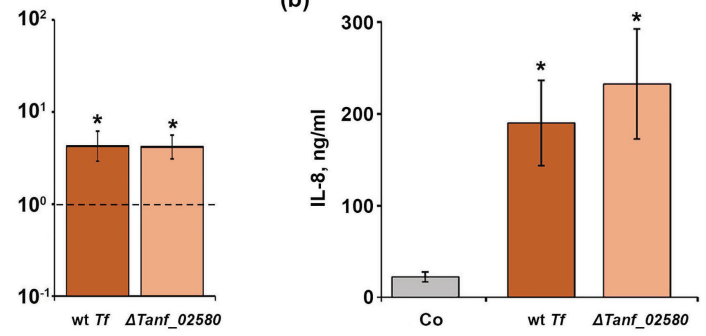

(c)

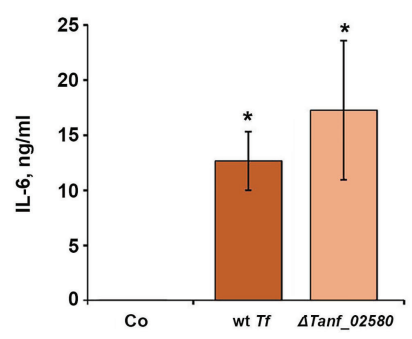

FIGURE 2 | Comparison of the effects of T. forsythia wild-type (wt Tf) and T9SS signal peptidase mutant ( $\Delta$ Tanf_02580) on the gene expression and protein production of TNF- $\alpha, \mathrm{IL}-8$, and IL-6 in U937 macrophages. (A) Macrophages were stimulated for $3 \mathrm{~h}$ (left panels) or $16 \mathrm{~h}$ (right panels) and the resulting expression of TNF- $\alpha$ (a), IL-8 (b), and IL-6 (c) was determined by qPCR. The $y$-axis shows n-fold expression of the target gene in relation to the unstimulated control ( $\mathrm{n}$-fold expression $=1$, indicated by the dashed line) determined by the $2^{-\Delta \Delta C t}$ method. IL-6 expression after $16 \mathrm{~h}$ was below the detection limit. (B) Macrophages were stimulated for $16 \mathrm{~h}$ and the resulting production of TNF- $\alpha$ (a), IL-8 (b), and IL-6 (c) was determined by ELISA. Non-stimulated cells served as a control (Co). All data are presented as mean \pm s.e.m of six independent experiments. * - Significantly different from control, with $P<0.05$. \# - Significantly different between two $T$. forsythia species, with $P<0.05$.

(Figure 5A). None of the proteins was detected in the conditioned media of $P$. gingivalis wild-type-stimulated hGFBs, whereas stimulation with the $\triangle P G 0026$ mutant resulted in significantly higher amounts of all secreted inflammatory mediators compared to the unstimulated control (Figure 5B).

\section{DISCUSSION}

The periodontal pathogens $T$. forsythia and P. gingivalis have developed ingenious strategies to evade host immune clearance and to exploit their pathogenic potential (Amano et al., 2014). These bacteria direct a specific class of their proteins, namely those equipped with a CTD, to the T9SS-a translocon unique for Bacteroidetes-to display their harmful or self-protecting cargo at the cell surface or secrete it into the exterior environment (Veith et al., 2009; de Diego et al., 2016). Various virulence factors have been demonstrated to be targeted to the T9SS ensuring OM trafficking. In this study, we have analyzed if and how depletion of OM proteins channeled through this protein secretion system affects the immune response of human macrophages and hGFBs to $T$. forsythia and $P$. gingivalis.

By MS-shot-gun proteomics we first confirmed that the OM of T. forsythia and P. gingivalis T9SS mutants defective in secretion of CTD-protein due to deletion of the signal peptidase genes Tanf_02580 and PG0026, respectively, were depleted from known CTD-proteins, whereas these were present in the parent strains. In the case of T. forsythia, this finding corroborates a previous analysis showing that the major CTD-proteins (TfsA and TfsB) of this bacterium forming the Slayer accumulated in the periplasm and the mutant lacked the Slayer (Tomek et al., 2014). Similarly, sortase mutants of $P$. gingivalis retained inactive gingipains in the periplasm (Mizgalska et al., 2021). Therefore, TfsA/TfsB and BspA surface antigen as well as the RgpA-, RgpB-, Kgp-gingipains from $P$. gingivalis served as leads for the assessment of the 
A
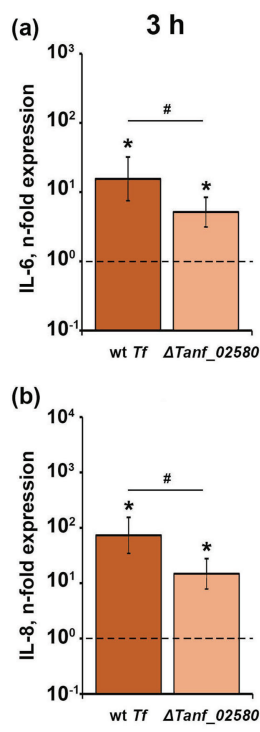

(c)

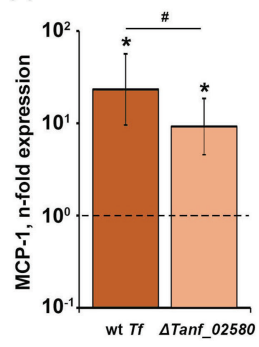

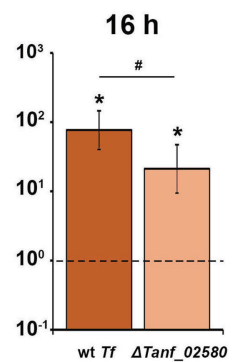
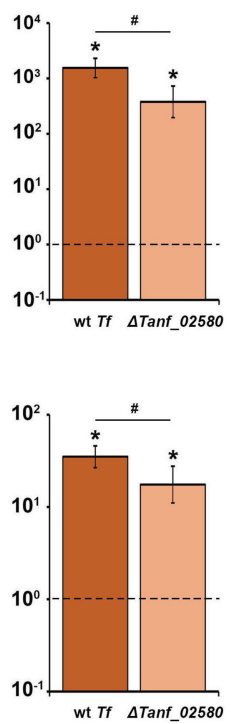

(a)

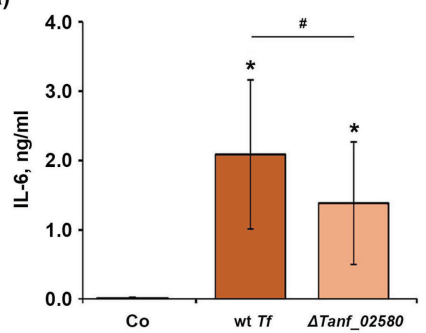

(b)

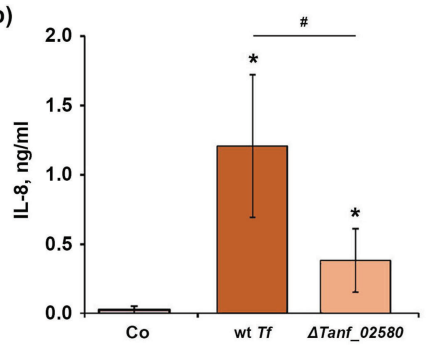

(c)

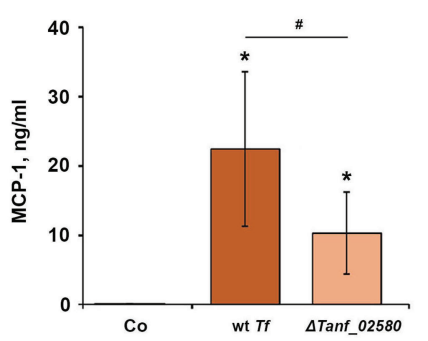

FIGURE 3 | Comparison of the effects of T. forsythia wild-type (wt Tf) and T9SS signal peptidase mutant ( $\Delta$ Tanf_02580) on the gene expression and protein production of TNF- $\alpha$, IL-8, and IL- 6 in hGFBs. (A) hGFBs were stimulated for $3 \mathrm{~h}$ (left panels) or $16 \mathrm{~h}$ (right panels) and the resulting expression of IL-6 (a), IL-8 (b), and MCP-1 (c) was determined by qPCR. The $y$-axis shows $n$-fold expression of the target gene in relation to the unstimulated control ( $\mathrm{n}$-fold expression $=1$, indicated by the dashed line) determined by the $2^{-\Delta \Delta \mathrm{Ct}}$ method. (B) hGFBs were stimulated for $16 \mathrm{~h}$ and the resulting production of IL-6 (a), IL-8 (b), and MCP-1 (c) was determined by ELISA. Non-stimulated cells served as a control (Co). All data are presented as mean \pm s.e.m of six independent experiments. ${ }^{\star}-$ Significantly different from control, with $P<0.05$. \# - Significantly different between two T. forsythia species, with $P<0.05$.

efficiency of T9SS shut-down in the signal peptidase mutants (Tables 1 and 2).

The viability of both human cell types included in this study upon bacterial infection was proven by the MTT assay, which is based on the measurements of mitochondrial cell activity (Figure 1). We found that at the tested MOI none of the bacterial species had a cytotoxic effect on the viability of macrophages and hGFBs (Figure 1). All bacteria induced an increase in the metabolic activity of macrophages, while this was not observed for hGFBs. The reason for this difference as well as the physiological importance of the increased viability of U937 macrophages after infection with $T$. forsythia and $P$. gingivalis is not entirely clear. Increased viability of macrophages might be due to the metabolic remodeling of these cells after bacterial infection (Fleetwood et al., 2017).

Shut-down of the T9SS in both T. forsythia and $P$. gingivalis altered the cytokine and chemokine response of host cells to these pathogens. The T9SS-deficient T. forsythia mutant $\Delta$ Tanf_02580 generally induced a lower inflammatory response compared to the wild-type species in hGFBs as shown by qPCR and ELISA (Figures $\mathbf{2}$ and $\mathbf{3}$ ). A similar tendency was observed in U937 macrophages. As shown by proteomic analysis, shut-down of the T9SS in T. forsythia inhibits the secretion of several proteins, and particularly the S-layer proteins TfsA and TfsB (Tomek et al., 2014) as well as BspA, which have opposite effects on the host response. On the one hand, the T. forsythia S-layer was shown to delay the host response at the early phase of infection (Sekot et al., 2011); consequently, its absence might result in higher cytokine production by the host cells. On the other hand, BspA is known to be a strong TLR-2 agonist (Hajishengallis et al., 2002; Onishi et al., 2008). Both U937 and hGFBs express TLR-2 and produce inflammatory cytokines upon the stimulation with TLR-2 agonists (Greene et al., 2004; Behm et al., 2020) and, therefore, the impairment of BspA secretion results in a lower host response. Since we have observed a lower inflammatory host response to the $\Delta$ Tanf_02580 mutant compared to the T. forsythia wild-type, we conclude that abolishing BspA OM translocation has a more 
A

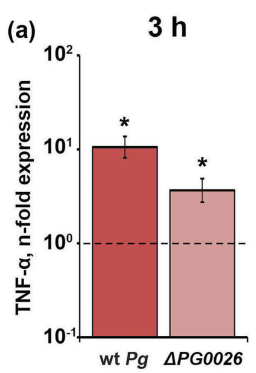

(b)

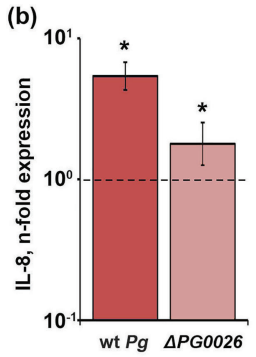

(c)

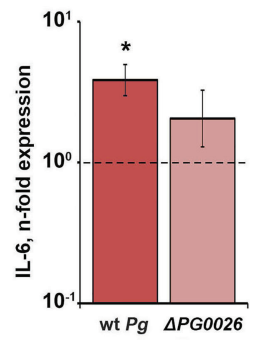

$16 \mathrm{~h}$
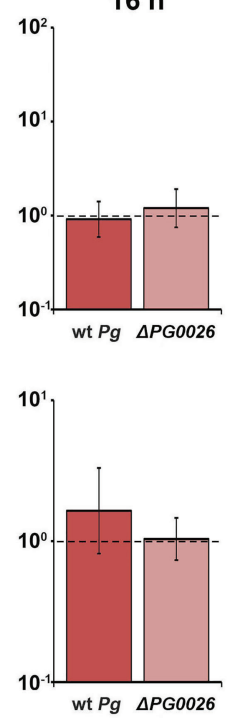

wt Pg $\triangle P G 0026$

B

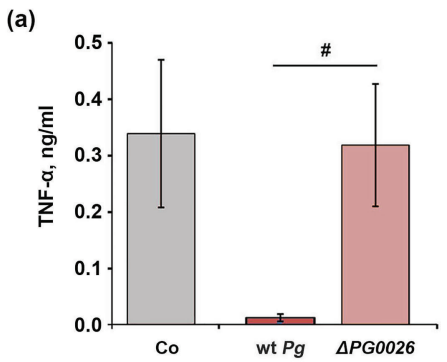

(b)

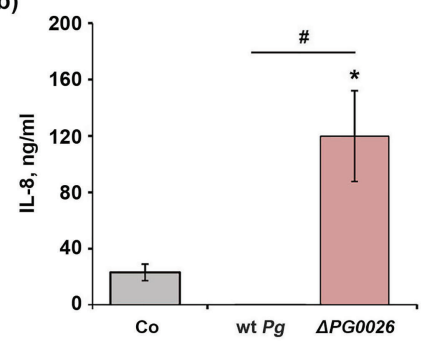

(c)

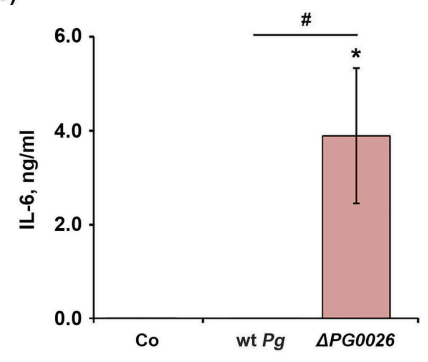

FIGURE 4 | Comparison of the effects of $P$. gingivalis wild-type (wt $P g$ ) and T9SS signal peptidase mutant ( $\Delta P G 0026)$ on the gene expression and protein production of TNF- $\alpha$, IL-8, and IL-6 in U937 macrophages. (A) Macrophages were stimulated for 3 hours (left panels) or 16 hours (right panels) and the resulting expression of TNF- $\alpha$ (a), IL-8 (b), and IL-6 (c) was determined by qPCR. The $y$-axis shows n-fold expression of the target gene in relation to the unstimulated control ( $\mathrm{n}$-fold expression $=1$, indicated by the dashed line) determined by the $2^{-\Delta \Delta \mathrm{Ct}}$ method. IL- 6 expression after $16 \mathrm{~h}$ was below the detection limit. (B) Macrophages were stimulated for $16 \mathrm{~h}$ and the resulting production of TNF- $\alpha$ (a), IL-8 (b), and IL-6 (c) was determined by ELISA. Non-stimulated cells served as a control (Co). All data are presented as mean \pm s.e.m of six independent experiments. ${ }^{*}-$ Significantly different from control, with $P<0.05$. \# - Significantly different between two $P$. gingivalis species, with $P<0.05$.

profound effect on the secretion of the tested inflammatory mediators. Quantitative differences between macrophages and hGFBs might be explained by the generally different responses of these cell types to various pathogen-associated molecular patterns. Particularly, our previous studies showed that the activation of the TLR-2 pathway induces a strong response in hGFBs and periodontal ligament cells, which is markedly higher than that induced by the TLR-4 agonist lipopolysaccharide (Andrukhov et al., 2016; Blufstein et al., 2019). Therefore, elimination of the secretion of the TLR-2 agonist BspA in the $\Delta$ Tanf_02580 mutant would strongly diminish the response of this cell type to $T$. forsythia.

Compared to $T$. forsythia, the deletion of the T9SS signal peptidase in $P$. gingivalis had a qualitatively strikingly different effect on the host response. $P$. gingivalis wild-type induced generally higher gene expression of TNF- $\alpha$, IL-8, and IL-6 in U937 macrophages three hours post stimulation compared to the $\triangle P G 0026$ mutant. However, the content of all investigated proteins in conditioned media was not increased after stimulation with $P$. gingivalis wild-type. Moreover, the levels of most of the investigated proteins after 3 hours were below the detection limit of the ELISA kit. In contrast, after stimulation with the $\triangle P G 0026$ mutant, a significantly increased amount of IL- 6 and IL- 8 was detected in the supernatant of U937 macrophages. This might be explained by the high activity of the $P$. gingivalis gingipains, which in fact are major virulence factors of this bacterium (Genco et al., 1999; Bao et al., 2014; O'Brien-Simpson et al., 2016). Gingipains have been shown to activate the host response by inducing secretion of IL- 6 by oral epithelial cells (Lourbakos et al., 2001), of IL- 8 by gingival fibroblasts (Oido-Mori et al., 2001), and of IL-6, IL-8 and MCP-1 in monocytic cells (Uehara et al., 2008). This is reflected by the slightly lower gene expression levels observed after stimulation with the T9SS-deficient mutant $\triangle P G 0026$ when 
A
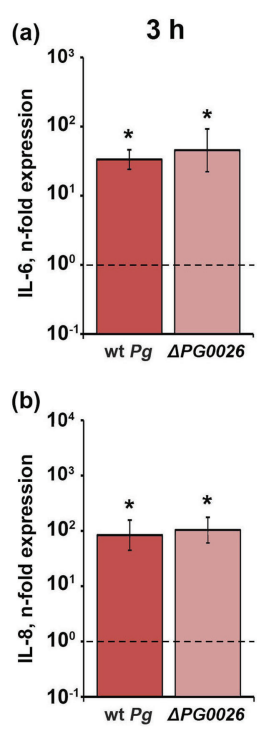

(c)

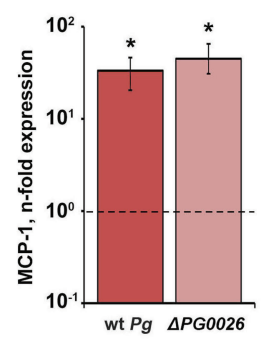

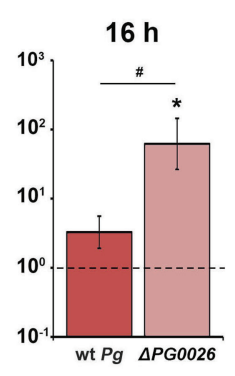
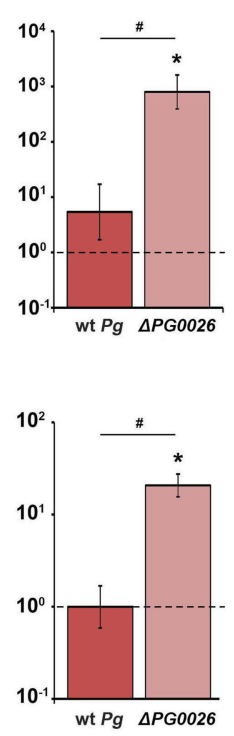

B

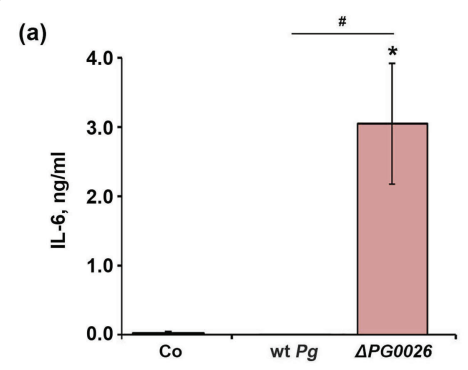

(b)

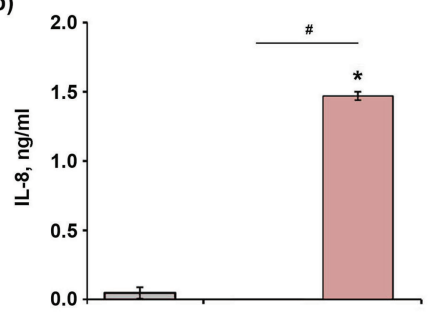

(c)

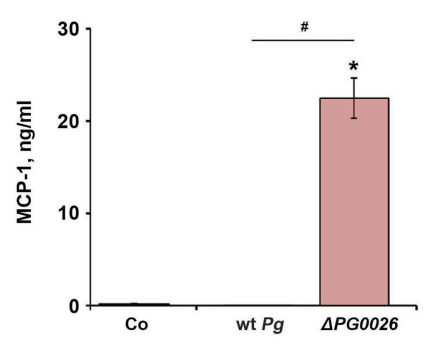

FIGURE 5 | Comparison of the effects of $P$. gingivalis wild-type (wt $P g$ ) and T9SS signal peptidase mutant $(\Delta P G 0026)$ on the gene expression and protein production of TNF- $\alpha$, IL-8, and IL-6 in hGFBs. (A) hGFBs were stimulated for 3 hours (left panels) or 16 hours (right panels) and the resulting expression of IL- 6 (a), IL-8 (b), and MCP-1 (c) was determined by qPCR. The $y$-axis shows $n$-fold expression of the target gene in relation to the unstimulated control ( $\mathrm{n}$-fold expression = 1 , indicated by the dashed line) determined by the $2^{-\Delta \Delta C t}$ method. (B) hGFBs were stimulated for $16 \mathrm{~h}$ and the resulting production of IL-6 (a), IL-8 (b), and MCP-1 (c) was determined by ELISA. Non-stimulated cells served as a control (Co). All data are presented as mean \pm s.e.m of six independent experiments. * - Significantly different from control, with $P<0.05$. \# - Significantly different between two $P$. gingivalis species, with $P<0.05$.

compared to the wild-type. On the other hand, gingipains are potent in the proteolytic cleavage of various host proteins, including inflammatory mediators (Yun et al., 1999; Yun et al., 2002; Tam et al., 2009). Thus, the secretion of gingipains by $P$. gingivalis wild-type has two consequences for the host cells' response; they might stimulate the gene expression and at the same time degrade the secreted proteins. This assumption might explain the qualitative differences in gene and protein expression in the response of U937 macrophages to two P. gingivalis species.

In hGFBs, the effects of wild-type and T9SS-deficient $P$. gingivalis were slightly different compared to U937 macrophages (Figures 4, 5). After 3 hours, the response of hGFBs to both species was similar on the gene expression level, whereas 16 hours post stimulation, the response to the T9SS-deficient mutant was markedly higher than that to $P$. gingivalis wild-type. Gingipain activity of the wild-type might also explain this difference. The response to bacterial pathogens in hGFBs might be increased by autocrine mechanisms mediated by IL-1 $\beta$ and TNF- $\alpha$ production (Naruishi and Nagata, 2018). This autocrine loop might be disrupted by gingipains, which would then result in lower gene expression levels upon the stimulation with $P$. gingivalis wild-type. Furthermore, gingipains have been shown to facilitate $P$. gingivalis cell adhesion and invasion (Chen and Duncan, 2004; Boisvert and Duncan, 2008; Fitzpatrick et al., 2009). In gingival epithelial cells, intracellular $P$. gingivalis can suppress the production of IL-8, interferon- $\gamma$ induced protein 10 and TLR-2 and inhibit apoptosis (Hajishengallis and Lamont, 2014). Blocking gingipain secretion in the T9SS-deficient mutant might result in a reduced ability to invade hGFBs and, therefore, higher gene expression levels can be observed after stimulation with this strain after 16 hours when compared to cells infected with the wild-type.

When comparing the response of U937 macrophages and hGFBs to the different bacterial species, the different intrinsic properties of these cell types need to be considered. Macrophages are immune cells and their response to bacteria is transient - 
recovery follows the initial increase in the gene expression to the initial levels within 24 hours (Nau et al., 2002). In contrast, hGFBs are assumed to contribute to a sustained inflammation and do not exhibit a tolerance state after prolonged stimulation with bacterial components (Ara et al., 2009; Blufstein et al., 2018).

\section{CONCLUSIONS}

We demonstrated that the shut-down of OM translocation of CTD-proteins via the T9SS in T. forsythia and P. gingivalis causes an alteration of the host immune response to these pathogens, Considering our data and those from the literature, it is conceivable to assume that the decreased host response to the T. forsythia T9SS-signal peptidase mutant and the drastically changed host response to the $P$. gingivalis mutant in comparison to the respective parent strain are largely due to the impaired secretion of BspA and the S-layer proteins in the T. forsythia T9SS mutant and the activity of the gingipains in the $P$. gingivalis T9SS mutant, respectively. Blocking gingipain secretion in $P$. gingivalis seems to have a dual effect, associated with both activation and inactivation of the host response (Imamura et al., 2003).

The abrogation of the T9SS secretion system in periodontal pathogens can have both pro- and anti-inflammatory effects and, therefore, the contribution of the T9SS to the host-microbiome interaction in the oral cavity needs further clarification. In a subcutaneous chamber model of infection in mice, the shutdown of the T9SS in $P$. gingivalis resulted in lower inflammation and decreased systemic dissemination of infection (Benedyk et al., 2019). However, the overall role of the T9SS in human periodontitis needs to be investigated in future basic and clinical studies. This might unravel future strategies for the treatment of periodontitis and/or prophylaxis of periodontitis.

\section{DATA AVAILABILITY STATEMENT}

The original contributions presented in the study are included in the article/Supplementary Material. Further inquiries can be directed to the corresponding authors.

\section{REFERENCES}

Amano, A., Chen, C., Honma, K., Li, C., Settem, R. P., and Sharma, A. (2014). Genetic Characteristics and Pathogenic Mechanisms of Periodontal Pathogens. Adv. Dent. Res. 26, 15-22. doi: 10.1177/0022034514526237

Andrukhov, O., Andrukhova, O., Ozdemir, B., Haririan, H., Muller-Kern, M., Moritz, A., et al. (2016). Soluble CD14 Enhances the Response of Periodontal Ligament Stem Cells to P. gingivalis Lipopolysaccharide. PloS One 11, e0160848. doi: 10.1371/journal.pone.0160848

Ara, T., Kurata, K., Hirai, K., Uchihashi, T., Uematsu, T., Imamura, Y., et al. (2009). Human Gingival Fibroblasts are Critical in Sustaining Inflammation in Periodontal Disease. J. Periodontal Res. 44, 21-27. doi: 10.1111/j.16000765.2007.01041.x

Baggiolini, M., and Clark-Lewis, I. (1992). Interleukin-8, a Chemotactic and Inflammatory Cytokine. FEBS Lett. 307, 97-101. doi: 10.1016/0014-5793(92) 80909-Z

\section{ETHICS STATEMENT}

The procedure for the isolation of hGFBs from the gingival tissue was approved by the Ethic Committee of the Medical University of Vienna (EK 1694/2015, extended in 2019). All patients gave their written consent. The patients/participants provided their written informed consent to participate in this study.

\section{AUTHOR CONTRIBUTIONS}

Conceptualization, CS and OA. Methodology, CS and OA. Validation, CS, OA, SB, and CG-G. Formal analysis, MB, MT, $\mathrm{SB}, \mathrm{CG}-\mathrm{G}, \mathrm{PN}, \mathrm{CS}$, and OA. Investigation, MB, MT, SB, CG-G, and $\mathrm{PN}$. Writing-original draft preparation, $\mathrm{MB}, \mathrm{OA}$, and CS. Writing-review and editing, MT, SB, JP, OA, and CS. Supervision, CS and OA. Funding acquisition, CS. All authors have read and agreed to the published version of the manuscript.

\section{FUNDING}

This research was funded by Austrian Science Fund FWF, projects P24317-B22 and P33618-B22 (to CS) and the FWF Doctoral Programme "BioToP - Biomolecular Technology of Proteins" W1224. OA was supported by Austrian Science Fund FWF, project P29440. JP was supported by NIH/NIDCR grants DE026280 and DE030939. Open Access Funding by the Austrian Science Fund (FWF). The funders had no role in the design of the study; in the collection, analyses, or interpretation of data; in the writing of the manuscript, or in the decision to publish the results.

\section{ACKNOWLEDGMENTS}

The authors thank Franziska Stritzinger for assistance with the immunological assays.

\section{SUPPLEMENTARY MATERIAL}

The Supplementary Material for this article can be found online at: https:/www.frontiersin.org/articles/10.3389/fcimb.2022.835509/ full\#supplementary-material

Bao, K., Belibasakis, G. N., Thurnheer, T., Aduse-Opoku, J., Curtis, M. A., and Bostanci, N. (2014). Role of Porphyromonas gingivalis Gingipains in Multi-Species Biofilm Formation. BMC Microbiol. 14, 258. doi: 10.1186/s12866-014-0258-7

Behm, C., Blufstein, A., Abhari, S. Y., Koch, C., Gahn, J., Schäffer, C., et al. (2020). Response of Human Mesenchymal Stromal Cells From Periodontal Tissue to LPS Depends on the Purity But Not on the LPS Source. Mediators Inflamm. 2020, 8704896. doi: 10.1155/2020/8704896

Behm, C., Blufstein, A., Gahn, J., Noroozkhan, N., Moritz, A., Rausch-Fan, X., et al. (2019). Soluble CD14 Enhances the Response of Periodontal Ligament Stem Cells to Toll-Like Receptor 2 Agonists. Mediators Inflamm. 2019, 8127301. doi: 10.1155/2019/8127301

Benedyk, M., Marczyk, A., and Chruscicka, B. (2019). Type IX Secretion System is Pivotal for Expression of Gingipain-Associated Virulence of Porphyromonas gingivalis. Mol. Oral. Microbiol. 34, 237-244. doi: 10.1111/omi.12268

Blufstein, A., Behm, C., Gahn, J., Uitz, O., Naumovska, I., Moritz, A., et al. (2019). Synergistic Effects Triggered by Simultaneous Toll-Like Receptor-2 and -3 
Activation in Human Periodontal Ligament Stem Cells. J. Periodontol 90, 1190-1201. doi: 10.1002/JPER.19-0005

Blufstein, A., Behm, C., Nguyen, P. Q., Rausch-Fan, X., and Andrukhov, O. (2018). Human Periodontal Ligament Cells Exhibit No Endotoxin Tolerance Upon Stimulation With Porphyromonas gingivalis Lipopolysaccharide. J. Periodontal Res. 53, 589-597. doi: 10.1111/jre.12549

Boisvert, H., and Duncan, M. J. (2008). Clathrin-Dependent Entry of a Gingipain Adhesin Peptide and Porphyromonas gingivalis Into Host Cells. Cell Microbiol. 10, 2538-2552. doi: 10.1111/j.1462-5822.2008.01228.x

Bradford, M. M. (1976). A Rapid and Sensitive Method for the Quantitation of Microgram Quantities of Protein Utilizing the Principle of Protein-Dye Binding. Anal. Biochem. 72, 248-254. doi: 10.1006/abio.1976.9999

Cekici, A., Kantarci, A., Hasturk, H., and Van Dyke, T. E. (2014). Inflammatory and Immune Pathways in the Pathogenesis of Periodontal Disease. Periodontol 2000 64, 57-80. doi: 10.1111/prd.12002

Charlie-Silva, I., Klein, A., Gomes, J. M. M., Prado, E. J. R., Moraes, A. C., Eto, S. F., et al. (2019). Acute-Phase Proteins During Inflammatory Reaction by Bacterial Infection: Fish-Model. Sci. Rep. 9, 4776. doi: 10.1038/s41598-019-41312-z

Chen, T., and Duncan, M. J. (2004). Gingipain Adhesin Domains Mediate Porphyromonas gingivalis Adherence to Epithelial Cells. Microb. Pathogen 36, 205-209. doi: 10.1016/j.micpath.2003.12.001

de Diego, I., Ksiazek, M., Mizgalska, D., Koneru, L., Golik, P., Szmigielski, B., et al. (2016). The Outer-Membrane Export Signal of Porphyromonas gingivalis Type IX Secretion System (T9SS) is a Conserved C-Terminal Beta-Sandwich Domain. Sci. Rep. 6, 23123. doi: 10.1038/srep23123

Deshmane, S. L., Kremlev, S., Amini, S., and Sawaya, B. E. (2009). Monocyte Chemoattractant Protein-1 (MCP-1): An Overview. J. Interfern Cytokine Res. 29, 313-326. doi: 10.1089/jir.2008.0027

Fitzpatrick, R. E., Wijeyewickrema, L. C., and Pike, R. N. (2009). The Gingipains: Scissors and Glue of the Periodontal Pathogen, Porphyromonas gingivalis. Fut Microbiol. 4, 471-487. doi: 10.2217/Fmb.09.18

Fleetwood, A. J., Lee, M. K. S., Singleton, W., Achuthan, A., Lee, M. C., O’BrienSimpson, N. M., et al. (2017). Metabolic Remodeling, Inflammasome Activation, and Pyroptosis in Macrophages Stimulated by Porphyromonas gingivalis and Its Outer Membrane Vesicles. Front. Cell Infect. Microbiol. 7, 351. doi: 10.3389/fcimb.2017.00351

Friedrich, V., Gruber, C., Nimeth, I., Pabinger, S., Sekot, G., Posch, G., et al. (2015). Outer Membrane Vesicles of Tannerella forsythia: Biogenesis, Composition, and Virulence. Mol. Oral. Microbiol. 30, 451-473. doi: 10.1111/omi.12104

Genco, C. A., Potempa, J., Mikolajczyk-Pawlinska, J., and Travis, J. (1999). Role of Gingipains R in the Pathogenesis of Porphyromonas gingivalis-Mediated Periodontal Disease. Clin. Infect. Dis. 28, 456-465. doi: 10.1086/515156

Gorasia, D. G., Glew, M. D., Veith, P. D., and Reynolds, E. C. (2020). Quantitative Proteomic Analysis of the Type IX Secretion System Mutants in Porphyromonas gingivalis. Mol. Oral. Microbiol. 35, 78-84. doi: 10.1111/ omi.12283

Greene, C. M., McElvaney, N. G., O'Neill, S. J., and Taggart, C. C. (2004). Secretory Leucoprotease Inhibitor Impairs Toll-Like Receptor 2- and 4-Mediated Responses in Monocytic Cells. Infect. Immun. 72, 3684-3687. doi: 10.1128/ iai.72.6.3684-3687.2004

Griffen, A. L., Beall, C. J., Campbell, J. H., Firestone, N. D., Kumar, P. S., Yang, Z. K., et al. (2012). Distinct and Complex Bacterial Profiles in Human Periodontitis and Health Revealed by 16 S Pyrosequencing. ISME J. 6, 11761185. doi: 10.1038/ismej.2011.191

Gundry, R. L., White, M. Y., Murray, C. I., Kane, L. A., Fu, Q., Stanley, B. A., et al. (2009). Preparation of Proteins and Peptides for Mass Spectrometry Analysis in a Bottom-Up Proteomics Workflow. Curr. Prot Mol. Biol. Chapter 10, Unit10.25-Unit10.25. doi: 10.1002/0471142727.mb1025s88

Hajishengallis, G., and Lamont, R. J. (2012). Beyond the Red Complex and Into More Complexity: The Polymicrobial Synergy and Dysbiosis (PSD) Model of Periodontal Disease Etiology. Mol. Oral. Microbiol. 27, 409-419. doi: 10.1111/ j.2041-1014.2012.00663.x

Hajishengallis, G., and Lamont, R. J. (2014). Breaking Bad: Manipulation of the Host Response by Porphyromonas gingivalis. Eur. J. Immunol. 44, 328-338. doi: $10.1002 /$ eji.201344202

Hajishengallis, G., Martin, M., Sojar, H. T., Sharma, A., Schifferle, R. E., DeNardin, E., et al. (2002). Dependence of Bacterial Protein Adhesins on Toll-Like
Receptors for Proinflammatory Cytokine Induction. Clin. Diagn. Lab. Immunol. 9, 403-411. doi: 10.1128/cdli.9.2.403-411.2002

Holt, S. C., and Ebersole, J. L. (2005). Porphyromonas gingivalis, Treponema denticola, and Tannerella forsythia: The "Red Complex", a Prototype Polybacterial Pathogenic Consortium in Periodontitis. Periodontol 2000 38, 72-122. doi: 10.1111/j.1600-0757.2005.00113.x

Imamura, T., Travis, J., and Potempa, J. (2003). The Biphasic Virulence Activities of Gingipains: Activation and Inactivation of Host Proteins. Curr. Protein Pept. Sci. 4, 443-450. doi: 10.2174/1389203033487027

Komatsuzawa, H., Asakawa, R., Kawai, T., Ochiai, K., Fujiwara, T., Taubman, M. A., et al. (2002). Identification of Six Major Outer Membrane Proteins From Actinobacillus actinomycetemcomitans. Gene 288, 195-201. doi: 10.1016/ s0378-1119(02)00500-0

Koneru, L., Ksiazek, M., Waligorska, I., Straczek, A., Lukasik, M., Madej, M., et al. (2017). Mirolysin, a LysargiNase From Tannerella forsythia, Proteolytically Inactivates the Human Cathelicidin, LL-37. Biol. Chem. 398, 395-409. doi: 10.1515/hsz-2016-0267

Ksiazek, M., Mizgalska, D., Eick, S., Thøgersen, I. B., Enghild, J. J., and Potempa, J. (2015). KLIKK Proteases of Tannerella forsythia: Putative Virulence Factors With a Unique Domain Structure. Front. Microbiol. 6, 312. doi: 10.3389/ fmicb.2015.00312

Laemmli, U. K. (1970). Cleavage of Structural Proteins During the Assembly of the Head of Bacteriophage T4. Nature 227, 680-685. doi: 10.1038/227680a0

Lasica, A. M., Goulas, T., Mizgalska, D., Zhou, X., de Diego, I., Ksiazek, M., et al. (2016). Structural and Functional Probing of PorZ, an Essential Bacterial Surface Component of the Type-IX Secretion System of Human OralMicrobiomic Porphyromonas gingivalis. Sci. Rep. 6, 37708. doi: 10.1038/ srep37708

Lasica, A. M., Ksiazek, M., Madej, M., and Potempa, J. (2017). The Type IX Secretion System (T9SS): Highlights and Recent Insights Into its Structure and Function. Front. Cell Infect. Microbiol. 7, 215. doi: 10.3389/fcimb.2017.00215

Lauber, F., Deme, J. C., Lea, S. M., and Berks, B. C. (2018). Type 9 Secretion System Structures Reveal a New Protein Transport Mechanism. Nature 564, 77-82. doi: 10.1038/s41586-018-0693-y

Lee, S. W., Sabet, M., Um, H. S., Yang, J., Kim, H. C., and Zhu, W. (2006). Identification and Characterization of the Genes Encoding a Unique Surface (S-) Layer of Tannerella forsythia. Gene 371, 102-111. doi: 10.1016/ j.gene.2005.11.027

Lourbakos, A., Potempa, J., Travis, J., D’Andrea, M. R., Andrade-Gordon, P., Santulli, R., et al. (2001). Arginine-Specific Protease From Porphyromonas gingivalis Activates Protease-Activated Receptors on Human Oral Epithelial Cells and Induces Interleukin-6 Secretion. Infect. Immun. 69, 5121-5130. doi: 10.1128/IAI.69.8.5121-5130.2001

Marsh and, Zaura (2011). Dental biofilm: ecological interactions in health and disease. J. Clin. Periodontol. 44 (Suppl 18), S12-S22. doi: 10.1111/jcpe.12679

Mishima, E., and Sharma, A. (2011). Tannerella forsythia Invasion in Oral Epithelial Cells Requires Phosphoinositide 3-Kinase Activation and ClathrinMediated Endocytosis. Microbiology 157, 2382-2391. doi: 10.1099/ mic.0.048975-0

Mizgalska, D., Goulas, T., Rodríguez-Banqueri, A., Veillard, F., Madej, M., Małecka, E., et al. (2021). Intermolecular Latency Regulates the Essential CTerminal Signal Peptidase and Sortase of the Porphyromonas gingivalis TypeIX Secretion System. Proc. Natl. Acad. Sci. U.S.A. 118. doi: 10.1073/ pnas. 2103573118

Murakami, Y., Higuchi, N., Nakamura, H., Yoshimura, F., and Oppenheim, F. G. (2002). Bacteroides forsythus Hemagglutinin is Inhibited by $\mathrm{N}$ Acetylneuraminyllactose. Oral. Microbiol. Immunol. 17, 125-128. doi: 10.1046/j.0902-0055.2001.00093.x

Naruishi, K., and Nagata, T. (2018). Biological Effects of Interleukin-6 on Gingival Fibroblasts: Cytokine Regulation in Periodontitis. J. Cell Physiol. 233, 63936400. doi: $10.1002 /$ jcp. 26521

Nau, G. J., Richmond, J. F., Schlesinger, A., Jennings, E. G., Lander, E. S., and Young, R. A. (2002). Human Macrophage Activation Programs Induced by Bacterial Pathogens. Proc. Natl. Acad. Sci. U.S.A. 99, 1503-1508. doi: 10.1073/ pnas. 022649799

Nguyen, K. A., Travis, J., and Potempa, J. (2007). Does the Importance of the CTerminal Residues in the Maturation of RgpB From Porphyromonas gingivalis 
Reveal a Novel Mechanism for Protein Export in a Subgroup of Gram-Negative Bacteria? J. Bacteriol 189, 833-843. doi: 10.1128/jb.01530-06

O’Brien-Simpson, N. M., Holden, J. A., Lenzo, J. C., Tan, Y., Brammar, G. C., Walsh, K. A., et al. (2016). A Therapeutic Porphyromonas gingivalis Gingipain Vaccine Induces Neutralising IgG1 Antibodies That Protect Against Experimental Periodontitis. NPJ Vaccines 1, 16022. doi: 10.1038/npjvaccines.2016.22

Oido-Mori, M., Rezzonico, R., Wang, P. L., Kowashi, Y., Dayer, J. M., Baehni, P. C., et al. (2001). Porphyromonas gingivalis Gingipain-R Enhances Interleukin-8 But Decreases Gamma Interferon-Inducible Protein 10 Production by Human Gingival Fibroblasts in Response to T-Cell Contact. Infect. Immun. 69, 44934501. doi: 10.1128/Iai.69.7.4493-4501.2001

Olsen, I., and Hajishengallis, G. (2016). Major Neutrophil Functions Subverted by Porphyromonas gingivalis. J. Oral. Microbiol. 8, 30936. doi: 10.3402/ jom.v8.30936

Onishi, S., Honma, K., Liang, S., Stathopoulou, P., Kinane, D., Hajishengallis, G., et al. (2008). Toll-Like Receptor 2-Mediated Interleukin-8 Expression in Gingival Epithelial Cells by the Tannerella forsythia Leucine-Rich Repeat Protein BspA. Infect. Immun. 76, 198-205. doi: 10.1128/iai.01139-07

Posch, G., Sekot, G., Friedrich, V., Megson, Z. A., Koerdt, A., Messner, P., et al. (2012). Glycobiology Aspects of the Periodontal Pathogen Tannerella forsythia. Biomolecules 2, 467-482. doi: 10.3390/biom 2040467

Rangarajan, M., Smith, S. J., U, S., and Curtis, M. A. (1997). Biochemical Characterization of the Arginine-Specific Proteases of Porphyromonas gingivalis W50 Suggests a Common Precursor. Biochem. J. 323 (Pt 3), 701709. doi: $10.1042 /$ bj3230701

Sabet, M., Lee, S.-W., Nauman, R. K., Sims, T., and Um, H.-S. (2003). The Surface (S-) Layer is a Virulence Factor of Bacteroides forsythus. Microbiology 149, 3617-3627. doi: 10.1099/mic.0.26535-0

Sakakibara, J., Nagano, K., Murakami, Y., Higuchi, N., Nakamura, H., Shimozato, K., et al. (2007). Loss of Adherence Ability to Human Gingival Epithelial Cells in S-Layer Protein-Deficient Mutants of Tannerella forsythensis. Microbiology 153, 866-876. doi: 10.1099/mic.0.29275-0

Sato, K., Yukitake, H., Narita, Y., Shoji, M., Naito, M., and Nakayama, K. (2013). Identification of Porphyromonas gingivalis Proteins Secreted by the Por Secretion System. FEMS Microbiol. Lett. 338, 68-76. doi: 10.1111/1574-6968.12028

Seers, C. A., Slakeski, N., Veith, P. D., Nikolof, T., Chen, Y. Y., Dashper, S. G., et al. (2006). The RgpB C-Terminal Domain has a Role in Attachment of RgpB to the Outer Membrane and Belongs to a Novel C-Terminal-Domain Family Found in Porphyromonas gingivalis. J. Bacteriol 188, 6376-6386. doi: 10.1128/jb.00731-06

Sekot, G., Posch, G., Messner, P., Matejka, M., Rausch-Fan, X., Andrukhov, O., et al. (2011). Potential of the Tannerella forsythia S-Layer to Delay the Immune Response. J. Dental Res. 90, 109-114. doi: 10.1177/0022034510384622

Sharma, A., Sojar, H. T., Glurich, I., Honma, K., Kuramitsu, H. K., and Genco, R. J. (1998). Cloning, Expression, and Sequencing of a Cell Surface Antigen Containing a Leucine-Rich Repeat Motif From Bacteroides forsythus ATCC 43037. Infect. Immun. 66, 5703-5710. doi: 10.1128/IAI.66.12.5703-5710.1998

Shimotahira, N., Oogai, Y., Kawada-Matsuo, M., Yamada, S., Fukutsuji, K., Nagano, K., et al. (2013). The Surface Layer of Tannerella forsythia Contributes to Serum Resistance and Oral Bacterial Coaggregation. Infect. Immun. 81, 1198-1206. doi: 10.1128/IAI.00983-12

Sochalska, M., and Potempa, J. (2017). Manipulation of Neutrophils by Porphyromonas gingivalis in the Development of Periodontitis. Front. Cell Infect. Microbiol. 7, 197. doi: 10.3389/fcimb.2017.00197

Socransky, S. S., and Haffajee, A. D. (2005). Periodontal Microbial Ecology. Periodontol 2000 38, 135-187. doi: 10.1111/j.1600-0757.2005.00107.x

Stathopoulou, P. G., Benakanakere, M. R., Galicia, J. C., and Kinane, D. F. (2009). The Host Cytokine Response to Porphyromonas gingivalis is Modified by Gingipains. Oral. Microbiol. Immunol. 24, 11-17. doi: 10.1111/j.1399302X.2008.00467.x

Stathopoulou, P. G., Benakanakere, M. R., Galicia, J. C., and Kinane, D. F. (2010). Epithelial Cell Pro-Inflammatory Cytokine Response Differs Across Dental
Plaque Bacterial Species. J. Clin. Periodontol 37, 24-29. doi: 10.1111/j.1600051X.2009.01505. $\mathrm{x}$

Tada, H., Shimizu, T., Matsushita, K., and Takada, H. (2017). Porphyromonas gingivalis-Induced IL-33 Down-Regulates hCAP-18/LL-37 Production in Human Gingival Epithelial Cells. BioMed. Res. 38, 167-173. doi: 10.2220/ biomedres.38.167

Tam, V., O'Brien-Simpson, N. M., Chen, Y. Y., Sanderson, C. J., Kinnear, B., and Reynolds, E. C. (2009). The RgpA-Kgp Proteinase-Adhesin Complexes of Porphyromonas gingivalis Inactivate the Th2 Cytokines Interleukin-4 and Interleukin-5. Infect. Immun. 77, 1451-1458. doi: 10.1128/IAI.01377-08

Tomek, M. B., Neumann, L., Nimeth, I., Koerdt, A., Andesner, P., Messner, P., et al. (2014). The S-Layer Proteins of Tannerella forsythia are Secreted via a Type IX Secretion System That is Decoupled From Protein O-Glycosylation. Mol. Oral. Microbiol. 29, 307-320. doi: 10.1111/omi.12062

Uehara, A., Imamura, T., Potempa, J., Travis, J., and Takada, H. (2008). Gingipains From Porphyromonas gingivalis Synergistically Induce the Production of Proinflammatory Cytokines Through Protease-Activated Receptors With Toll-Like Receptor and NOD1/2 Ligands in Human Monocytic Cells. Cell Microbiol. 10, 1181-1189. doi: 10.1111/j.1462-5822.2008.01119.x

Veith, P. D., O'Brien-Simpson, N. M., Tan, Y., Djatmiko, D. C., Dashper, S. G., and Reynolds, E. C. (2009). Outer Membrane Proteome and Antigens of Tannerella forsythia. J. Proteome Res. 8, 4279-4292. doi: 10.1021/pr900372c

Veith, P. D., Shoji, M., O’Hair, R. A. J., Leeming, M. G., Nie, S., Glew, M. D., et al. (2020). Type IX Secretion System Cargo Proteins are Glycosylated at the CTerminus With a Novel Linking Sugar of the Wbp/Vim Pathway. mBio 11. doi: $10.1128 / \mathrm{mBio} .01497-20$

Vistica, D. T., Skehan, P., Scudiero, D., Monks, A., Pittman, A., and Boyd, M. R. (1991). Tetrazolium-Based Assays for Cellular Viability: A Critical Examination of Selected Parameters Affecting Formazan Production. Cancer Res. 51, 2515-2520.

Wilensky, A., Tzach-Nahman, R., Potempa, J., Shapira, L., and Nussbaum, G. (2015). Porphyromonas gingivalis Gingipains Selectively Reduce CD14 Expression, Leading to Macrophage Hyporesponsiveness to Bacterial Infection. J. Innate Immun. 7, 127-135. doi: 10.1159/000365970

Yun, P. L. W., DeCarlo, A. A., Collyer, C., and Hunter, N. (2002). Modulation of an Interleukin-12 and Gamma Interferon Synergistic Feedback Regulatory Cycle of T-Cell and Monocyte Cocultures by Porphyromonas gingivalis Lipopolysaccharide in the Absence or Presence of Cysteine Proteinases. Infect. Immun. 70, 5695-5705. doi: 10.1128/Iai.70.10.5695-5705.2002

Yun, P. L. W., DeCarlo, A. A., and Hunter, N. (1999). Modulation of Major Histocompatibility Complex Protein Expression by Human Gamma Interferon Mediated by Cysteine Proteinase-Adhesin Polyproteins of Porphyromonas gingivalis. Infect. Immun. 67, 2986-2995. doi: 10.1128/Iai.67.6.2986-2995.1999

Conflict of Interest: The authors declare that the research was conducted in the absence of any commercial or financial relationships that could be construed as a potential conflict of interest.

Publisher's Note: All claims expressed in this article are solely those of the authors and do not necessarily represent those of their affiliated organizations, or those of the publisher, the editors and the reviewers. Any product that may be evaluated in this article, or claim that may be made by its manufacturer, is not guaranteed or endorsed by the publisher.

Copyright (c) 2022 Braun, Tomek, Grünwald-Gruber, Nguyen, Bloch, Potempa, Andrukhov and Schäffer. This is an open-access article distributed under the terms of the Creative Commons Attribution License (CC BY). The use, distribution or reproduction in other forums is permitted, provided the original author(s) and the copyright owner(s) are credited and that the original publication in this journal is cited, in accordance with accepted academic practice. No use, distribution or reproduction is permitted which does not comply with these terms. 